\title{
Topological defects in the edge-state structure in a bilayer electron system
}

\author{
E. V. Deviatov, ${ }^{1, *}$ V. T. Dolgopolov, ${ }^{1}$ A. Würtz, ${ }^{2}$ A. Lorke, ${ }^{2}$ A. Wixforth, ${ }^{3}$ W. Wegscheider, ${ }^{4}$ K. L. Campman, ${ }^{5}$ and \\ A. C. Gossard ${ }^{5}$ \\ ${ }^{1}$ Institute of Solid State Physics RAS, Chernogolovka, Moscow District 142432, Russia \\ ${ }^{2}$ Laboratorium für Festkörperphysik, Universität Duisburg-Essen, Lotharstrasse 1, D-47048 Duisburg, Germany \\ ${ }^{3}$ Institut für Physik, Universitat Augsburg, Universitatsstrasse, 1 D-86135 Augsburg, Germany \\ ${ }^{4}$ Institut für Angewandte und Experimentelle Physik, Universitat Regensburg, 93040 Regensburg, Germany \\ ${ }^{5}$ Materials Department and Center for Quantized Electronic Structures, University of California, Santa Barbara, California 93106, USA
}

(Received 18 March 2005; published 14 July 2005)

\begin{abstract}
We experimentally demonstrate formation of pointlike topological defects in the edge-state structure in the quantum Hall effect regime. By using of a selective population technique, we investigate equilibration processes between the edge states in bilayer electron structures with a high tunneling rate between layers. Unexpected flattening of the $I-V$ curves in a perpendicular magnetic field at a specific filling factor combination and the recovery of the conventional nonlinear $I-V$ characteristics in tilted fields give a strong evidence for the existence of topological defects.
\end{abstract}

DOI: $10.1103 /$ PhysRevB.72.041305

PACS number(s): 73.40.Qv, 71.30.+h

Since its report by Halperin, ${ }^{1}$ the concept of the edge states (ES) was found to be useful in describing transport phenomena in two-dimentional (2D) systems for both sharp $^{2}$ and smooth $^{3}$ edge potential profiles. ES arise in a quantizing magnetic field at the intersections of the Fermi level with distinct Landau levels, which are bent up by the edge potential.

Much attention was paid to substantiate the ES picture experimentally by investigating electron transport along the ES, as well as between them (for a review, see Ref. 4). Different imaging techniques ${ }^{5,6}$ were used to demonstrate the existence of compressible and incompressible strips of the electron liquid at the sample edge, which is widely accepted nowadays.

However, there is another class of phenomena in ES that are still open for investigation. It is the formation of socalled topological defects, which have been predicted to occur, e.g., when two ES with different spins locally switch their positions and thus cross each other at several (at least two) points. ${ }^{7,8}$ Possible mechanisms for such a crossover were proposed theoretically for nonequilibrium occupation of spin-split ES, when the chemical-potential difference is of the order of the spin splitting. ${ }^{8}$ This situation cannot be achieved in usual experiments performed in the Hall-bar geometry. ${ }^{9}$ In recent experiments in quasi-Corbino geometry, ${ }^{10,11}$ with direct biasing of the ES, no sign of ES crossing was observed, even when the ES imbalances exceeded the cyclotron gap. ${ }^{12}$ This may be due to the fact that even at the crossing points, the electron spin flips, which are necessary for the inter-ES scattering, can be strongly suppressed. ${ }^{9}$

More pronounced features may be expected in the transport properties of bilayer systems, where not only spin, but also isospin, related to the layer index, ${ }^{13}$ is involved. The energies of the isospin states can be tuned in situ by application of a suitable gate voltage, which, as we will show below, gives direct control over the presence of the topological defects.
Here, we experimentally study topological defects (namely, the edge-state crossings) in a bilayer electron system. We use a selective population technique to investigate equilibration processes between different edge states. By adjusting the gate voltage and the magnetic field, we force the bilayer system to be in different isospin states in the gated and ungated regions, that leads to a crossing of the ES, which we detect in the transport characteristics.

Measurements are performed in a quasi-Corbino sample geometry, in which a top gate of a special shape is used to independently contact the ES and bring them into interaction over a distance that is much smaller than the equilibration length. ${ }^{10}$ In Fig. 1(a), only the interaction region of the sample is shown. Voltage $V$, applied between contacts that are connected to the inner and outer ES (not shown in Fig. 1 ), produces an energy shift $e V(e<0)$ between them. This allows us to directly investigate the charge transfer between
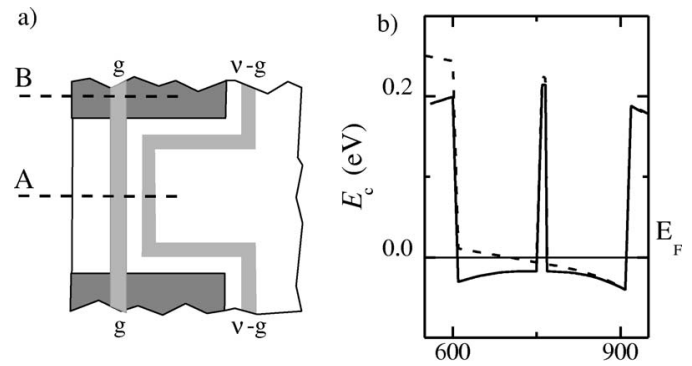

$z(\mathrm{~A})$

FIG. 1. (a) Schematic diagram of the gate-gap region in the pseudo-Corbino geometry. The shaded area represents the Schottky gate. In a quantizing magnetic field at total filling factor $\nu$, one set of the edge states (the number is equal to the filling factor under the gate, $g ; g<\nu$ ) is propagating under the gate along the etched edge of the sample, while the other edge states (their number is $\nu-g$ ) are going along the gate edge. In the gate-gap region, both sets of the edge states are running in parallel. (b) A quantum well subband diagram at zero gate voltage (solid line) and at smaller electron concentration, near bilayer onset (dashed line), is shown. 
ES in the interaction region (gate gap) at imbalances even higher than the spectral gaps.

In gated bilayer systems, such as the one with quasiCorbino geometry used here, the gate bias does not only influence the lateral, but also the vertical distribution of charge. At zero gate voltage, the bilayer systems studied here are slightly imbalanced (i.e., they have different electron concentrations in two parts of the well). A negative top-gate bias increases this initial imbalance [see Fig. 1(b)]. The electric field mostly affects the electron density in the top (closest to the gate) layer of the well, which will deplete at some bias $V_{\text {on }}$ (the bilayer onset voltage). At lower gate voltages, only the back part of the well is filled, so that the electron system becomes a single layer. Thus, even for a sample where the edge states in the gate gap originate from the bilayer spectrum, the channels under the gate may originate from either a bi- or a single-layer spectrum. By using samples with different $V_{\text {on }}$ it is therefore possible to realize different regimes under the gate, even at the same filling factor. For a single-layer system in a quantizing magnetic field, the energy spectrum is the usual Landau ladder of energy levels. In the bilayer regime, the situation is more sophisticated. ${ }^{14-17}$ In imbalanced bilayer systems, the electrons belong to a particular electron sheet, which makes it necessary to introduce a new quantum number: the isospin with projection equal to $\pm 1 / 2$ for top and/or bottom layers, correspondingly. Every isospin projection corresponds to an independent Landau ladder in the energy spectrum.

In sufficiently high magnetic fields (corresponding to low filling factors $\nu=1$ and 2), and/or in a balanced electron system, the situation is different. The electrons have nonzero wave functions in both layers and, consequently, are in a mixed isospin state. The energy spectrum is then a single Landau ladder with an additional energy gap: the symmetric-

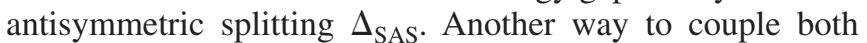
layers is through application of an in-plane magnetic field component. Not only does it increase the Zeeman splitting with respect to other energy scales, but it also forces common subband formation ${ }^{15}$ even at high filling factors $\nu>2$.

In the present paper we investigate the samples at filling factor $\nu=3$ in the gate gap and $g=1,2$ under the gate. We show below that the $I-V$ curve and its behavior in a tilted magnetic field is sensitive to the presence of topological defects in the ES structure.

In a perpendicular magnetic field at filling factor $\nu=3$, ES in the gate gap originate from two independent Landau ladders [see Fig. 2(a)]. The spatial separation between the two outer spin-split ES and the single inner one is given by the difference $\Gamma$ of the subband energies of the two electron layers in the bulk.

Let us first consider the case when the electron system under the gate is in a single-layer regime for filling factor $g=2$. Two spin-split quantum levels in the bottom electron layer form two ES, which are directly connected to the two outer spin-split ES in the gate gap, because the spin and isospin indices are the same [see Fig. 2(b)]. Applying a bias voltage to the ohmic contacts 1 and 2 in Fig. 2 produces an energy shift of the two outer ES in the gate gap with respect to the inner one. Positive voltage $V>0$ flattens the potential relief between the ES (Refs. 10 and 11) because of $e V<0$.

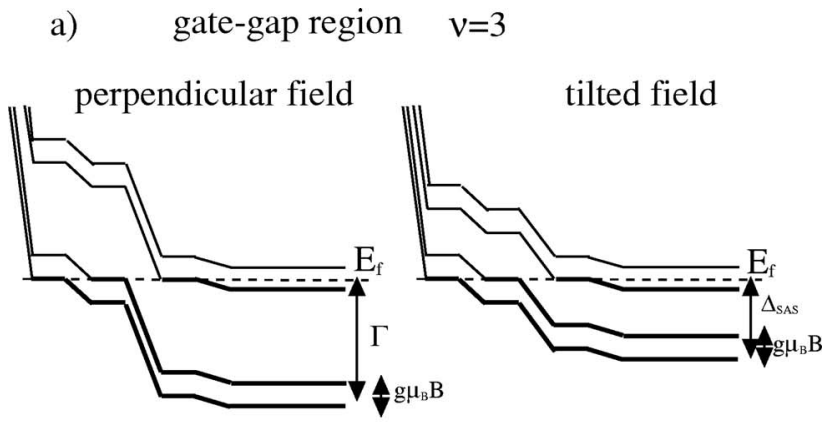

b) single-layer $\mathrm{g}=2$
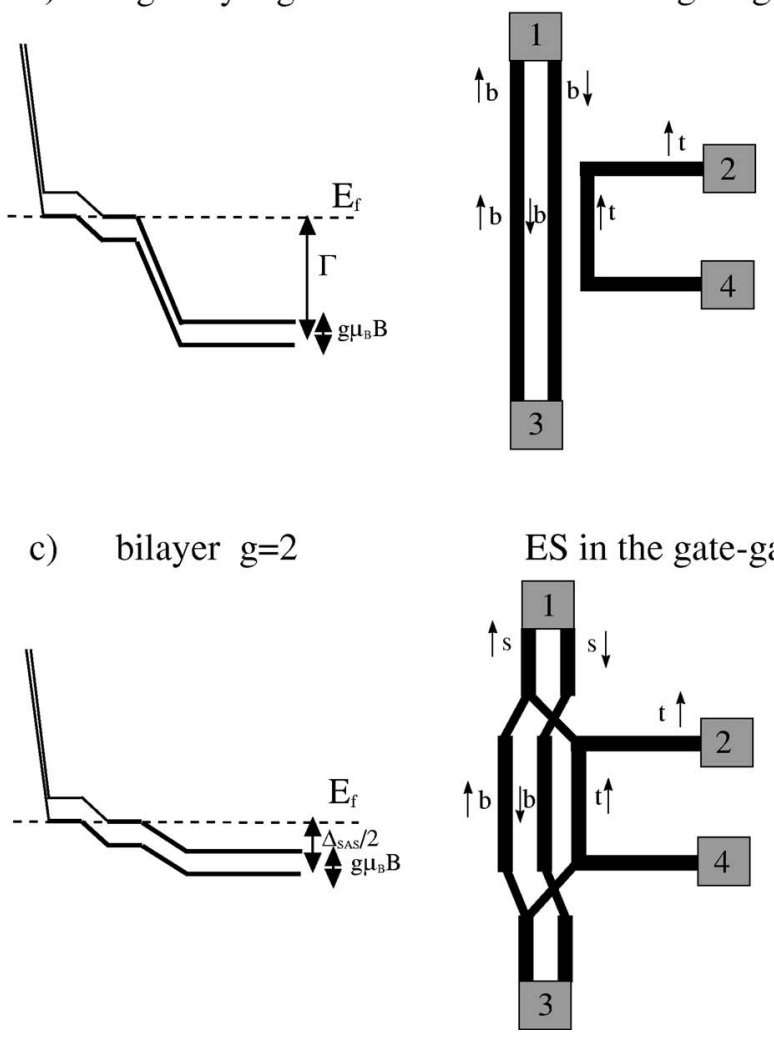

FIG. 2. Energy subband diagram of the sample edge for the filling factors $\nu=3$ and $g=2$ with no voltage applied between the ES: (a) in the gate-gap region in perpendicular and tilted magnetic fields; (b) under the gate in a single-layer regime (sample $B$ ) and the resulting ES structure in the gate gap; (c) under the gate in a bilayer regime (sample $A$ ) and the resulting ES structure in the gate gap. Symbols $t, b, s, \uparrow, \downarrow$ indicate the isospin (top, bottom, symmetric) and the spin (up and down) states of the ES. Ohmic contacts, connected to different ES, are denoted by gray bars.

Significant current starts to flow only after the complete flattening of the subband bottom, i.e., at $V_{\text {th }}^{+} \sim \Gamma$, so this branch of the $I-V$ curve should contain a clear-defined threshold. Applying a negative voltage $V<0$ produces only a tunnel current between $\mathrm{ES}$, leading to a strongly nonlinear $I-V$ branch.

Let us now turn to the case where the gated region is in the bilayer regime at $g=2$. Then the one-to-one correspondence between the edge states in the ungated and gated parts is no longer possible and a new ES topology will develop, as 
shown in Fig. 2(c), right. Under the gate, two quantum levels are filled. They both correspond to the lowest (symmetric) subband and are separated by the spin gap, see Fig. 2(c), left. Electrons in the corresponding ES belong to both electron layers simultaneously. Roughly speaking, there is a $50 \%$ probability to find an electron in either layer. When a charge is injected into the gate-gap region, both spin and isospin should be conserved. Isospin conservation means that approximately half of electrons are injected into the top electron layer and the others are injected into the bottom layer. Taking into account the spin conservation, we conclude that the electrons from the outer ES under the gate will be injected into both the outermost and the innermost ES in the gate gap, so they will share the same chemical potential. Thus, the ES can cross each other in the corners of the gate gap, as sketched in Fig. 2(c), right. Two ES with the same spin in the gate gap will be fully equilibrated, leading to a linear $I-V$ curve.

The described picture can easily be destroyed by an inplane magnetic field component. As mentioned above, in tilted magnetic fields the states in the gate-gap region will also be delocalized between both layers. As shown in Fig. 2(a), right, two spin-split ES will originate from the lowest (symmetric) quantum well state, while the third edge state will be associated with the antisymmetric state, separated by $\Delta_{S A S}$. Because the two outermost edge states now have the same isospin in both gated and ungated regions of the sample, the ES topology will resemble the one shown in Fig. 2(b), left. Therefore, there will be no ES crossing and the $I$ $-V$ curves should become strongly nonlinear again, as in the single-layer case. The height of the step on positive branch of $I-V$ curve will now be given by $\Delta_{S A S}$.

From the picture described above we expect that ES crossings can be realized in samples where the gated and ungated parts are in a different isospin configuration. The equilibration caused by the crossings should lead to a linear $I-V$ trace. These linear $I-V$ 's in normal magnetic fields and the recovery of the conventional nonlinear $I-V$ characteristics in tilted fields are the key features of the topological defects in bilayer systems.

Our samples are from two different wafers, $A$ and $B$, grown by molecular beam epitaxy on a semi-insulating $\mathrm{GaAs}$ substrate. They are GaAs/AlGaAs quantum wells of different widths and shapes. For wafer $A$, active layers form a $760-\AA$-wide parabolic quantum well, whereas for wafer $B$, a $300-\AA$-wide quantum well is of rectangular form. In the center of each well, a three-monolayer-thick $\mathrm{Al}_{x} \mathrm{Ga}_{1-x} \mathrm{As}(x=0.3)$ sheet is grown, which serves as a tunnel barrier. The symmetrically doped wells are capped by $600-\AA$ $\mathrm{Al}_{x} \mathrm{Ga}_{1-x} \mathrm{As}(x=0.3)$ and GaAs layers. Both structures, $A$ and $B$, contain a two-dimensional electron gas (2DEG) of similar electron concentration (about $4 \times 10^{11} \mathrm{~cm}^{-2}$ ) and mobility. They are slightly imbalanced (i.e., have unequal electron concentrations in two parts of the well).

The samples are patterned in a quasi-Corbino geometry. ${ }^{10}$ Ohmic contacts are made to both electron layers simultaneously. In the experiment we study the $I-V$ characteristics of the gate-gap region at the temperature of $30 \mathrm{mK}$ in magnetic fields up to $14 \mathrm{~T}$. Tilting the sample plane with respect to the magnetic field allows us to introduce an in-plane field com-

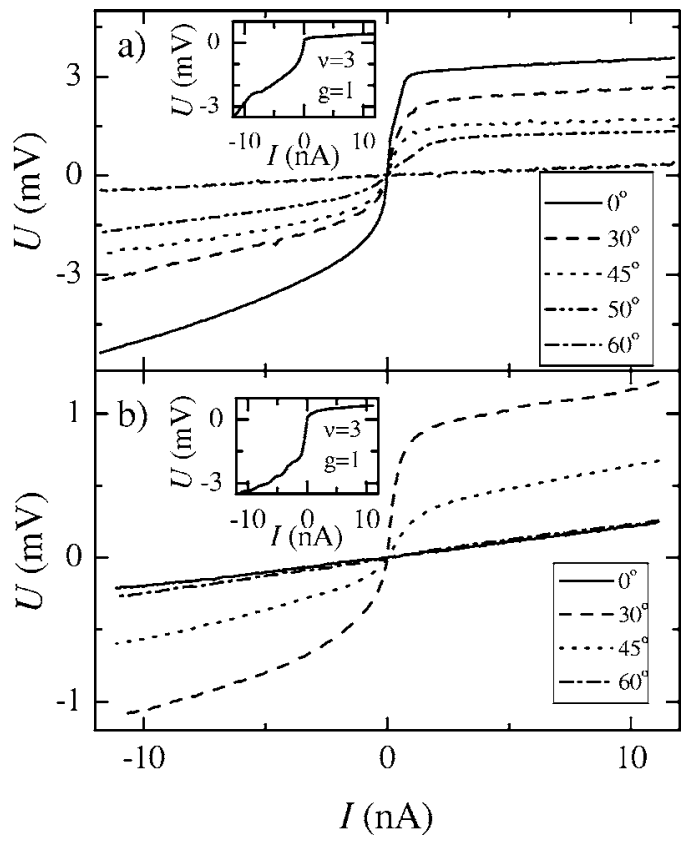

FIG. 3. $I$ - $V$ curves for samples $B$ (a) and $A$ (b) for filling factors $\nu=3$ and $g=2$ at different tilt angles equal to $\theta=0$ (solid line), $\theta=30^{\circ}$ (dashed line), $\theta=45^{\circ}$ (dotted line), $\theta=50^{\circ}$ [dash-dot-dot, only the (a) panel], $\theta=60^{\circ}$ (dash-dot line). A perpendicular magnetic field is constant and is equal to $6.6 \mathrm{~T}$ for the sample $B$ and 5.9 $\mathrm{T}$ for the sample $A$. The insets demonstrate $I-V$ curves for samples $B$ and $A$ at filling factors $\nu=3$ and $g=1$ in a perpendicular magnetic field.

ponent to affect the energy spectrum of the bilayers. The electron concentration in the ungated region was obtained from the usual magnetoresistance measurements. Also, magnetocapacitance measurements were performed to determine the energy spectrum under the gate. ${ }^{14}$

The onset voltage $V_{\text {on }}$, at which the system becomes single layer, is very different for both samples: $V_{\text {on }}=-0.3 \mathrm{~V}$ for the wafer $A$ and $V_{\text {on }}=-0.12 \mathrm{~V}$ for the wafer $B$. Therefore, at the filling factor combination $\nu=3, g=2$ that we are interested in here, the samples are in different isospin configurations. For sample $A, g=2$ is obtained at $V_{g}=-0.19>V_{\text {on }}$, so that the sample is in a bilayer regime. Sample $B$, on the other hand, is single layer at $g=2\left(V_{g}=-0.15<V_{\text {on }}\right)$. Both samples are in an equivalent isospin state at the filling factor combination $\nu=3, g=1\left(V_{g}<V_{\text {on }}\right.$, resulting in a single layer under the gate), which can be used as a reference.

In Fig. 3, $I-V$ curves are presented for both types of the samples, in normal and tilted magnetic fields. For the sample that is single layer at $g=2$ [Fig. 3(a)], the $I-V$ curves are strongly nonlinear. They have a well-defined threshold at positive voltages $(\sim 3 \mathrm{meV}$ in a perpendicular field), above which a current starts to flow. The value of the threshold corresponds to the subband splitting.

In contrast, the sample with bilayer $g=2$ [A-type, Fig. $3(\mathrm{~b})]$ has a fully linear $I-V$ curve in perpendicular magnetic fields. This demonstrates the absence of a gap between the inner and outer edge states, in agreement with the ES picture discussed above [Fig. 2(c), right]. Tilting the sample, while keeping the normal field component constant, causes the 
$I-V$ curve to become strongly nonlinear. This is in agreement with the prediction that the in-plane field component will remove the topological defects and reestablish a gap structure [see Fig. 2(a), right].

Further increase of the tilt angle flattens the $I-V$ curves, similarly for both sample types. It is important that even at the highest tilt angle $\theta=60^{\circ}, I-V$ 's for both samples are slightly nonlinear, while in a perpendicular field, the curve is fully linear for the sample of type $A$.

The conclusion that the different behaviors of the two samples shown in Fig. 3 are related to the different isospin configuration is further supported by measuring the $I-V$ characteristics at the filling factor combination $\nu=3, g=1$. In this case, both samples are in the same isospin configuration, and the curves are qualitatively similar for both samples in perpendicular (see insets to Fig. 3), as well as in tilted fields (not shown here). They are nonlinear and asymmetric, with a small positive threshold voltage given by the spin gap.

As mentioned above, the linear $I-V$ curve of sample $A$ for the filling factor combination $\nu=3, g=2$ in a perpendicular magnetic field indicates full equilibration between the inner and any (or both) of two outer ES in the gate-gap region. Figure 2(c), right, suggests that the innermost ES will only interact with the outermost ES (but not the middle one) if the spin conservation is assumed. We find that the experimental
$I-V$ slope exactly coincides with one obtained from Buttiker's calculation ${ }^{11}$ if the equilibration in the gate-gap is established between two of the ES involved. This allows us to draw the following conclusions: (i) because the gate-gap width $(2 \mu \mathrm{m})$ is much smaller than the characteristic equilibration lengths in transport between the ES (of the order of $100 \mu \mathrm{m}$; see Refs. 4 and 9, a defect must be present that very efficiently couples different ES; (ii) this defect only couples ES with the same spin in the gate gap; (iii) the equilibration by the defect is destroyed by an in-plane field component. These experimental findings, combined together, give strong evidence that the defect is not an impurity of some kind, but indeed a topological defect induced by the different isospin configurations in the gated and ungated regions of the sample.

We wish to thank A.A. Shashkin and Yu.A. Melnikov for help during the experiment. We also thank Greg Snider for the Poisson-Schrodinger solver. We gratefully acknowledge financial support by the RFBR, RAS, the Program "The State Support of Leading Scientific Schools," Deutsche Forschungsgemeinschaft, and SPP "Quantum Hall Systems," under Grant No. LO 705/1-2. E.V.D. acknowledges support by Russian Science Support Foundation.
*Corresponding author. Electronic address: dev@issp.ac.ru

${ }^{1}$ B. I. Halperin, Phys. Rev. B 25, 2185 (1982).

${ }^{2}$ M. Büttiker, Phys. Rev. B 38, 9375 (1988).

${ }^{3}$ D. B. Chklovskii, B. I. Shklovskii, and L. I. Glazman, Phys. Rev. B 46, 4026 (1992).

${ }^{4}$ R. J. Haug, Semicond. Sci. Technol. 8, 131 (1993).

${ }^{5}$ A. A. Shashkin, A. J. Kent, J. R. Owers-Bradley, A. J. Cross, P. Hawker, and M. Henini, Phys. Rev. Lett. 79, 5114 (1997).

${ }^{6}$ Y. Y. Wei, J. Weis, K. v. Klitzing, and K. Eberl, Phys. Rev. Lett. 81, 1674 (1998).

${ }^{7}$ Jed Dempsey, B. Y. Gelfand, and B. I. Halperin, Phys. Rev. Lett. 70, 3639 (1993); B. Y. Gelfand and B. I. Halperin, Phys. Rev. B 49, 1862 (1994).

${ }^{8}$ Lex Rijkels and Gerrit E. W. Bauer, Phys. Rev. B 50, 8629 (1994)

${ }^{9}$ G. Müller, D. Weiss, A. V. Khaetskii, K. von Klitzing, S. Koch, H. Nickel, W. Schlapp, and R. Lösch, Phys. Rev. B 45, 3932 (1992).

${ }^{10}$ A. Würtz, R. Wildfeuer, A. Lorke, E. V. Deviatov, and V. T.
Dolgopolov, Phys. Rev. B 65, 075303 (2002).

${ }^{11}$ E. V. Deviatov, V. T. Dolgopolov, A. Wurtz, JETP Lett. 79, 618 (2004).

${ }^{12}$ E. V. Deviatov, A. Wurtz, A. Lorke, M. Yu. Melnikov, V. T. Dolgopolov, D. Reuter, A. D. Wieck, Phys. Rev. B 69, 115330 (2004).

${ }^{13}$ L. Zheng, R. J. Radtke, and S. Das Sarma, Phys. Rev. Lett. 78, 2453 (1997).

${ }^{14}$ V. T. Dolgopolov, A. A. Shashkin, E. V. Deviatov, F. Hastreiter, M. Hartung, A. Wixforth, K. L. Campman, and A. C. Gossard, Phys. Rev. B 59, 13235 (1999).

${ }^{15}$ E. V. Deviatov, V. S. Khrapai, A. A. Shashkin, V. T. Dolgopolov, F. Hastreiter, A. Wixforth, K. L. Campman, and A. C. Gossard, JETP Lett. 71, 496 (2000).

${ }^{16}$ A. Sawada, Z. F. Ezawa, H. Ohno, Y. Horikoshi, Y. Ohno, S. Kishimoto, F. Matsukura, M. Yasumoto, and A. Urayama, Phys. Rev. Lett. 80, 4534 (1998)

${ }^{17}$ A. G. Davies, C. H. W. Barnes, K. R. Zolleis, J. T. Nicholls, M. Y. Simmons, and D. A. Ritchie, Phys. Rev. B 54, R17331 (1996). 